\title{
Biofilm and productivity-associated community changes in serial- transfer experiments in heterogeneous liquid microcosms
}

\author{
Robyn Jerdan, Emily Donaldson, Scott Cameron, and Andrew Spiers \\ Abertay University, School of Applied Sciences, United Kingdom of Great Britain and Northern Ireland (r.jerdan@abertay.ac.uk)
}

Static incubation of liquid microcosms results in a physically heterogeneous environment, where depletion of $\mathrm{O}_{2}$ in the lower region creates a relatively high- $\mathrm{O}_{2}$ niche directly below the air-liquid (A$\mathrm{L}$ ) interface. This has been investigated using the model bacterium Pseudomonas fluorescens SBW25 and the biofilm-forming adaptive mutant known as the Wrinkly Spreader. In this system, colonisation of the A-L interface by the Wrinkly Spreader provides a fitness advantage over nonbiofilm-forming competitors, including the ancestral SBW25, due to better access to $\mathrm{O}_{2}$ in an otherwise $\mathrm{O}_{2}$-growth limiting environment. Our current research seeks to understand how the ecological interactions of this simple system applies in more complex communities, where biofilms can be produced by multiple competing or co-operative strains and the low $-\mathrm{O}_{2}$ region colonised by a range of strains capable of micro-aerobic growth. Here we report the effect of selection on the productivity of A-L interface biofilm-forming communities initiated by soil-wash (SW) inocula, which were serially transferred across ten microcosms and sixty days with mixed-community or biofilmonly samples. Initial analysis of the serial transfer experiments shows a decrease in community productivity which is explained by the accumulation of toxic metabolites, though small increases in community biofilm strength and attachment were also observed. Isolate-level analysis revealed a decrease in community diversity and a biofilm-associated phenotypic shift between the SW inocula and final-transfer communities, and these changes provide evidence of selection within our system.

Cell-localisation experiments confirm enrichment at the top of the liquid column in the high- $\mathrm{O}_{2}$ region, but also show high cell densities in the low- $\mathrm{O}_{2}$ region, even within the biofilm-only finaltransfer communities. Samples taken from the biofilm and lower region of these communities were able to re-colonise both in fresh microcosms, indicating that community members were capable of migration within the liquid column. Despite the over-all decrease seen in community productivity in the serial transfer experiments, we suggest that communities maximised productivity by colonising both regions of the liquid column, with a resource trade-off between fast growth in the highly competitive high $-\mathrm{O}_{2}$ region and slower growth in the less-competitive low- $\mathrm{O}_{2}$ region. Many isolates from the final-transfer communities could occupy both regions and were capable of migration, with almost all isolates capable of flagella-mediated motility, and we interpret this ability to move between regions as a fitness advantage in A-L interface biofilm-forming communities. Although we have not been able to test this directly using the final-transfer communities or isolates, we have been able to demonstrate a fitness advantage in the less complex $P$. fluorescens SBW25 system, where biofilm-forming mutants capable of colonising both regions had a greater competitive fitness advantage over those with a poor ability to colonise the liquid column.

How to cite: Jerdan, R., Donaldson, E., Cameron, S., and Spiers, A.: Biofilm and productivityassociated community changes in serial-transfer experiments in heterogeneous liquid microcosms, biofilms 9 conference, Karlsruhe, Germany, 29 September-1 Oct 2020, biofilms9-17, https://doi.org/10.5194/biofilms9-17, 2020 